\title{
Model building in almost-commutative geometry
}

\section{Christoph Stephan*}

Universität Potsdam, Institut für Mathematik

E-mail: christophstephan@gmx.de

\begin{abstract}
Alain Connes' noncommutative geometry allows to unify the classical Yang-Mills-Higgs theory and General relativity in a single geometrical framework, so called almost-commutative geometries. This unification implies restrictions for the couplings of the Standard Model at a given cut-off energy which reduce the degrees of freedom compared to the classical Standard Model. I will give an introduction to the basic ideas of almost-commutative model building and present models beyond the Standard Model that may be phenomenologically interesting. These models include extensions of the fermionic and the gauge sector as well as extensions of the scalar sector.
\end{abstract}

Frontiers of Fundamental Physics 14 - FFP14,

15-18 July 2014

Aix Marseille University (AMU) Saint-Charles Campus, Marseille

${ }^{*}$ Speaker. 